\title{
Umbilical cord-derived mesenchymal stem cell conditioned medium reverses neuronal oxidative injury by inhibition of TRPM2 activation and the JNK signaling pathway
}

Xueyun Liang ( $\sim$ liangxy2104@hotmail.com )

General Hospital of Ningxia Medical University https://orcid.org/0000-0002-4735-4418

Yan Wang

General Hospital of Ningxia Medical University

Jiaxin Liu

General Hospital of Ningxia Medical University

Baocong Yu

Ningxia Medical University

Yiran Jin

General Hospital of Ningxia Medical University

Jiahui Li

General Hospital of Ningxia Medical University

Xiaona Ma

General Hospital of Ningxia Medical University

Jianqiang $\mathrm{Yu}$

Ningxia Medical University

Jianguo Niu

Ningxia Medical University

\section{Research Article}

Keywords: mesenchymal stem cells, conditioned medium, TRPM2, neuronal apoptosis, MAPK

Posted Date: March 3rd, 2022

DOI: https://doi.org/10.21203/rs.3.rs-1383797/v1

License: (c) (1) This work is licensed under a Creative Commons Attribution 4.0 International License.

Read Full License 


\section{Umbilical cord-derived mesenchymal stem cell conditioned medium reverses neuronal oxidative injury by inhibition of TRPM2 activation and the JNK signaling pathway}

Yan Wang ${ }^{\# 1}$; Jiaxin $\mathrm{Liu}^{\# 1}$; Baocong $\mathrm{Yu}^{\# 2}$; Yiran $\mathrm{Jin}^{1} ; \mathrm{Jiahui}^{1}{ }^{1}$; Xiaona Ma ${ }^{1}$; Jianqiang $\mathrm{Yu}^{* 3}$; Jianguo Niu*2; Xueyun Liang*1

${ }^{1}$ Key Laboratory of Ningxia Stem Cell and Regenerative Medicine, General Hospital of Ningxia Medical University, Yinchuan, 750001, China

${ }^{2}$ Ningxia Key Laboratory of Cerebrocranial Diseases, Ningxia Medical University, Yinchuan, 750004, China;

${ }^{3}$ School of Pharmacology, Ningxia Medical University, Yinchuan, 750004, China.

\# These authors have contributed equally to this work and share first authorship.

* Corresponding authors: Jianqiang Yu, yujq910315@163.com;

Jianguo Niu, niujg2008@hotmail.com;

Xueyun Liang, liangxy2104@hotmail.com; 


\section{Abstract}

In this study, we aimed to clarify the protective effect of umbilical cord-derived mesenchymal stem cell conditioned medium (UC-MSC-CM) on neuronal oxidative injury and its potential mechanism. Neuronal oxidative damage was mimicked by $\mathrm{H} 2 \mathrm{O} 2$ treatment of the HT22 cell line. Immunofluorescence staining was used to analyze the expression of cleaved Caspase 3 and TRPM2. Cytotropic factors secreted by UC-MSCs were analyzed by enzymelinked immunosorbent assay. Whole-cell patch clamping was used to detect TRPM2-like currents. Western blotting was utilized to analyze the protein levels of SOD, p-ERK1/2, pJNK1/2/3 and cleaved-Caspase-9. The number of cleaved-Caspase-3-positive cells and protein expression of Caspase-9 induced by $\mathrm{H} 2 \mathrm{O} 2$ treatment were decreased by UC-MSC-CM treatment. Furthermore, SOD protein expression was increased in the MSC-CM group compared with that in the $\mathrm{H} 2 \mathrm{O} 2$ group. The H2O2-induced TRPM2-like currents in HT22 cells were attenuated by MSC-CM treatment. In addition, $\mathrm{H} 2 \mathrm{O} 2$ treatment downregulated the expression of p-JNK protein in HT22 cells, and this the downward trend was reversed by incubation with MSC-CM. Thus, we showed that UC-MSC-CM protects neurons against oxidative injury, possibly by inhibiting activation of TRPM2 and the JNK signaling pathway. Key Words: mesenchymal stem cells, conditioned medium, TRPM2, neuronal apoptosis, MAPK

22

(1)

25




\section{Introduction}

Stroke is the second leading cause of death worldwide and a major contributor to disability. Ischemic stroke, which is the most common type of this event[1], disrupts the production and clearance of reactive oxygen species (ROS), leading to their accumulation in brain tissue. This, in turn, results in the destruction or changes in lipids, proteins and nucleic acids in a cascade reaction that ultimately causes neuronal death[2]. However, no effective therapeutic strategies have yet been developed to prevent or treat oxidative stress injury.

Mesenchymal stem cells (MSCs) have been shown to exert antioxidant functions in experimental animals[3]. In recent years, MSCs have been widely used in experimental investigations of nerve injury repair because of their strong regeneration potential and ability to improve the microenvironment by secreting a variety of cytokines[4]. MSC-conditioned medium (MSC-CM) is heterogeneous and contains various soluble factors that can reduce lipid peroxidases generation, while promoting antioxidant enzyme production[5-8]. However, the mechanism by which MSC-CM protects neuronal cells against ischemic injury remains to be elucidated.

Transient receptor potential melatonin 2 (TRPM2) belongs to the TRP family and is a nonselective cation channel that allows the entry of calcium ions[9-11]. Studies have shown that $\mathrm{H}_{2} \mathrm{O}_{2}$ and ROS activate TRPM2 to induce the accumulation of calcium ions in cells, leading to cell death[12]. Importantly, recent studies have shown that TRPM2 channels play an important role in ischemic stroke, with TRPM2 activation further aggravating the condition[13-15].

In this study, we investigated the protective effect of umbilical cord-derived MSC-CM on $\mathrm{H}_{2} \mathrm{O}_{2}$-induced neuronal injury, and the potential roles of TRPM2 inhibition and the MAPK pathway in the underlying mechanism.

\section{Materials and Methods}

\subsection{HT22 cell culture}

HT22 cells were purchased from Bena Chuang Lian Biotechnology Co., Ltd (Beijing, China) .and cultured in Dulbecco's Modified Eagle Medium (DMEM; Gibco, USA) containing $5 \%$ fetal bovine serum (FBS; Biological Industries, Israel) and incubated at $37^{\circ} \mathrm{C}$ under $5 \%$ $\mathrm{CO}_{2}$ in a humidified atmosphere.

\subsection{Preparation of human umbilical cord-MSC-CM}


This study was approved by the Ethics Committee of the General Hospital of Ningxia Medical University, China. Human umbilical cord-MSCs (UC-MSCs) were isolated from the umbilical cord connective tissue collected from healthy patients after obtaining informed consent. $3 \times 10^{5}$ UM-MSCs were seeded in $100 \mathrm{~mm}$ culture plate containing Ultra Culture Serum-free Medium (Lonza, Switzerland) supplemented with 2\% Pall Ultroser G Serum Substitute (Pall, USA), and cultured in the $\mathrm{CO}_{2}$ incubator maintained at $37^{\circ} \mathrm{C}$ with $5 \% \mathrm{CO}_{2}$ and a humidified atmosphere. Medium was changed every 3 days. At approximately $90 \%$ confluence, cells were passaged and $3 \times 10^{5} \mathrm{UM}-\mathrm{MSC}$ reseeded in a new $100 \mathrm{~mm}$ culture plate. $1 \times 10^{6}$ UC-MSCs at passages 4 were seeded in a new $100 \mathrm{~mm}$ culture plate. At $60 \%-70 \%$ confluence, the medium was changed and the cells cultured for a further $24 \mathrm{~h}$ before harvesting the conditioned medium (CM). The CM was filtered $(0.22-\mu \mathrm{m}$ pore size) to remove cellular debris and concentrated using ultrafiltration units with a $3-\mathrm{kDa}$ molecular-weight cutoff (Millipore, Burlington, MA) to obtain the MSC-CM.

\subsection{Experimental design}

HT22 cells were divided into the following experimental groups: Normal: culture in complete medium; $\mathrm{H}_{2} \mathrm{O}_{2}$ : treated with $100 \mu \mathrm{M} \mathrm{H}_{2} \mathrm{O}_{2}$ for $1 \mathrm{~h}$; MSC-CM: administration of UCMSC-CM after treatment with $100 \mu \mathrm{M} \mathrm{H}_{2} \mathrm{O}_{2}$ for $1 \mathrm{~h}$; SP600125 (a JNK inhibitor) and PD98059 (an ERK inhibitor): starved with $0.5 \%$ serum for $6 \mathrm{~h}$, and cultured in the presence of the inhibitors for $24 \mathrm{~h}$ (SP600125 $50 \mu \mathrm{M}$; PD98059 $60 \mu \mathrm{M}$; MCE, USA); SP600125/PD98059 $+\mathrm{H}_{2} \mathrm{O}_{2}$ : cultured in the presence of the inhibitors for $24 \mathrm{~h}$ before the addition of $100 \mu \mathrm{M} \mathrm{H}_{2} \mathrm{O}_{2}$ for $1 \mathrm{~h}$; SP600125/PD98059 + MSC-CM: cultured in the presence of the inhibitors for $24 \mathrm{~h}$ before the addition of $100 \mu \mathrm{M} \mathrm{H}_{2} \mathrm{O}_{2}$ for $1 \mathrm{~h}$, when UC-MSCs-CM were added.

\subsection{Enzyme-linked immunosorbent assay (ELISA)}

Cystatin SA, CD200 R1, Pax3, HIF-1beta, Neuroligin2, FGF-21, HGF, VEGF, BDNF, EGF, GDNF and NT3 were detected in MSC-CM and Fibroblast-CM using commercial ELISA kits (Cloud-Clone Corp, China) according to the manufacturer's instructions.

\subsection{Whole-cell patch clamp experiment}

HT22 cells were cultured on Matrigel coated cover slides in 35mm culture dishes containing DMEM supplemented with 5\% FBS. The prepared HT22 cell slides were recorded in the 
whole-cell mode at room temperature. The cells were perfused continuously with DMEM supplemented with 5\% FBS. The pipette solution contained $(\mathrm{mM})$ : 125 potassium D-gluconate, $8 \mathrm{NaCl}, 2 \mathrm{Mg}$ ATP, 0.3 Na GTP, 0.2 EGTA and 10 HEPES, with the pH adjusted to 7.2-7.4 using $\mathrm{KOH}$. The recording glass electrodes were pulled from the borosilicate glass using a P97 laser electrode puller (Sutter Instruments Company, USA), and the resistance between the electrode tip and cell membrane was $8-12 \mathrm{M} \Omega$. Cells were held at a potential of $-60 \mathrm{mV}$ and current-voltage relations were obtained from voltage ramps from -80 to $+80 \mathrm{mV}$ (50 ms duration). Membrane currents were digitally sampled at a frequency of $10 \mathrm{kHz}$ and low-pass filtered at $2-5 \mathrm{kHz}$. $\mathrm{H}_{2} \mathrm{O}_{2}(100 \mu \mathrm{M}$; Yantai, China) was used to activate the TRPM2 channels. Data acquisition was performed using EPC-10 Patch Clamp Amplifier (HEKA Elektronik, Germany). The results were analyzed and plotted using GraphPad Prism software, version 7.04 .

\subsection{Immunofluorescence staining}

HT22 cells were fixed for 10 min with $4 \%$ paraformaldehyde (Biotopped Life Sciences, China), washed with PBS and permeabilized with $0.5 \%$ Triton-X in PBS for $1 \mathrm{~h}$ at room temperature. Non-specific binding was reduced by incubation with blocking buffer $(0.5 \%$ Triton-X in PBS+5\% normal goat serum) for $1 \mathrm{~h}$ at room temperature. Cells were then incubated overnight at $4{ }^{\circ} \mathrm{C}$ with the following primary antibodies diluted in blocking buffer: anti-Caspase3 (1:1,000, cat. no. ab49822; Abcam, UK), anti-TRPM2 (1:500, cat. no. ab11168; Abcam). Cells were then incubated for $3 \mathrm{~h}$ at room temperature with the following fluorescently-labeled secondary antibody diluted in blocking buffer: Cy3-labeled goat antirabbit (1:1,000, cat. no 111-165-003; Jackson ImmunoResearch Laboratories, USA). Cells were incubated with $4^{\prime}, 6^{\prime}$ diamino-2-phenylindole dihydrochloride (DAPI) (1:4; Solarbio, China) for 3-5 $\mathrm{min}$ at room temperature and washed with PBS before collecting images under a fluorescence microscope (Olympus, Japan) in the dark.

\subsection{Western blot analysis}

Total proteins were extracted from the HT22 cells using IP Lysis buffer (cat. no 87787; Thermo, USA) with protease and phosphatase inhibitors (cat. no. 78441; Thermo, USA). The concentration was determined using the bicinchoninic acid (BCA) method (cat. no. P0012; Beyotime, China). Equal amounts of protein samples were separated by sodium dodecyl sulfate-polyacrylamide gel electrophoresis (SDS-PAGE) with $10 \%$ and $15 \%$ gels (cat. no. PG112 and PG114; Epizyme Biotechnology, China) and transferred to polyvinylidene fluoride 
membranes (Millipore, Burlington, MA, USA). The membranes were blocked with 5\% nonfat milk for $2 \mathrm{~h}$ and incubated overnight at $4^{\circ} \mathrm{C}$ with the following primary antibodies: antiSOD (1:1,000, cat. no. ab183881; Abcam), anti-pJNK1/2/3 (1:1,000, cat. no. ab219584; Abcam), anti-pERK1/2(1:1,000, cat. no. ab201015; Abcam) and anti-Caspase9 (1:1,000, cat. no. WL01551; Wanleibio, China), and anti- $\beta$-actin (1:10,000, cat. no. 66009-1-Ig; Proteintech, USA). The membranes were then incubated for $1 \mathrm{~h}$ at room temperature with the following secondary antibodies: IRDye680RD goat anti-rabbit (1:20,000, cat. no. 926-68071; Licor, USA), IRDye680RD goat anti-mouse (1:20,000, cat. no. 926-68180; Licor) and IRDye800CW goat anti-mouse (1:20,000, cat. no. 926-32351; Licor). The signal on the membrane were visualized by the Odyssey infrared scanner (Licor, USA) and optical densities were quantified by Image $\mathrm{J}$ software. $\beta$-actin was used as a loading control.

\subsection{Statistical analysis}

Statistical analysis was performed using GraphPad Prism software, version 7.04. Data are presented as mean \pm standard deviation (SD) of at least three experiments. One-way analysis of variance (ANOVA) and repeated measures ANOVA were used for multivariate data analyses. $P<0.05$ was set as the threshold for statistical significance.

\section{Results}

\subsection{UC-MSC-CM protects $\mathrm{HT} 22$ cells against $\mathrm{H}_{2} \mathrm{O}_{2}$-induced neuronal apoptosis}

To examine the protective ability of UC-MSC-CM, we treated $\mathrm{H}_{2} \mathrm{O}_{2}$-stimulated HT22 cells with UC-MSC-CM for $24 \mathrm{~h}$. Compared with the $\mathrm{H}_{2} \mathrm{O}_{2}$-treated group, the density of $\mathrm{HT} 22$ cells was higher after treatment with UC-MSC-CM, although there was no morphological difference between the cells in the two groups $(P<0.05$, Fig. 1A). Fluorescence immunostaining showed that the number of Caspase-3 positive cells was significantly lower in the UC-MSCs-CM group compared with that in $\mathrm{H}_{2} \mathrm{O}_{2}$-treated group $(P<0.05$, Fig. $1 \mathrm{~B}$ and C). Furthermore, Western blot analysis showed that the expression level of cleaved-Caspase9 protein was significantly lower in the UC-MSC-CM group compared with that in $\mathrm{H}_{2} \mathrm{O}_{2}$ treated group $(P<0.05$, Fig. 1D and $\mathrm{E})$.

3.2 UC-MSC-CM is composed of cellular growth factors and mediates anti-oxidative functions 
The cytotropic factors secreted into the UC-MSC-CM were analyzed by ELISA. Many growth factors, such as EGF, BDNF, GDNF and HGF, were detected in the UC-MSCs-CM (Fig. 2A). Western blot analysis showed that the expression level of SOD protein was significantly higher in UC-MSC-CM group compared with that in the $\mathrm{H}_{2} \mathrm{O}_{2}$-treated group $(P$ $<0.05$, Fig. 2B and C).

\subsection{UC-MSC-CM suppresses TRPM2-related currents}

TRPM2 can be activated by $\mathrm{H}_{2} \mathrm{O}_{2}$-induced oxidative stress[16]. Fluorescence immunostaining confirmed the expression of TRPM2 in HT22 cells, with no marked differences in the number of TRPM2-positive cells and the cellular location of TRPM2 between the groups (Fig. 3A). Whole-cell patch clamp measurement were showed that $\mathrm{H}_{2} \mathrm{O}_{2}$ stimulation of HT22 cells evoked a non-selective inward current with current-voltage properties similar to those that are characteristic of TRPM2[17]. This $\mathrm{H}_{2} \mathrm{O}_{2}$-induced TRPM2like current in HT22 cells was attenuated by treatment with UC-MSC-CM (Fig. 3B)

\subsection{The neuroprotective effects of UC-MSC-CM are mediated via the JNK signaling} pathway

To explore the role of the $\mathrm{H}_{2} \mathrm{O}_{2}$-related MAPKs signaling pathway in the mechanism by which UC-MSC-CM protects HT22 cells against $\mathrm{H}_{2} \mathrm{O}_{2}$-induced injury, we analyzed the expression of p-JNK, p-P38 and p-ERK proteins. Western blot analysis showed that the expression level of p-JNK protein was significantly decreased after treatment with $\mathrm{H}_{2} \mathrm{O}_{2}$ and that this trend was reversed by subsequent treatment with UC-MSC-CM $(P<0.05$, Fig. 4A and B) accompanied by a decreased in the expression level of cleaved-Caspase 9 protein $(P<0.05$, Fig. 4C and D). The expression level of p-ERK protein was also decreased after treatment with $\mathrm{H}_{2} \mathrm{O}_{2}$, and the levels were unchanged by subsequent treatment with UC-MSC-CM (Fig.4D and E). The protein expression of p-P38 has not been detected in each group (data not shown).

\section{Discussion}

Under conditions of cerebral ischemia, the excitotoxicity and subsequent ROS overproduction can damage neuronal structures, leading to cell death. Many studies have indicated that MSC transplantation is a promising therapy for cerebral ischemia. To date, several studies have evaluated the neuroprotective effects of MSC-CM in various diseases and many showed positive results[18]. Pre-clinically, MSC-CM has been shown to exert cellular protective and regenerative effects[19]. Recently, MSC-CM has been identified as one of the 
key components of the neuroprotective mechanisms of MSCs and can effectively ameliorate ischemia/reperfusion-induced brain injury by promoting angiogenesis, regulating immune responses, and inhibiting neuronal apoptosis [20, 21]. In accordance with other reports, we provided further evidence for MSC-CM treatment as a potential approach to the preservation of neuronal integrity and function. Our study showed that the MSC-CM treatment protected HT22 cells from $\mathrm{H}_{2} \mathrm{O}_{2}$-induced oxidative injury by decreasing apoptosis and increasing the cellular anti-oxidative ability.

Cerebral ischemia-induced TRPM2 activation triggers abnormal intracellular $\mathrm{Ca}^{2+}$ accumulation and cell death, which in turn causes irreversible brain damage[22]. Thus, TRPM2 has emerged as a new therapeutic target for ischemic stroke. TRPM2 inhibitors have been in preclinical development to prevent pathological $\mathrm{Ca}^{2+}$ overload[23-25]; however, successful development of specific inhibitors with good pharmacokinetic properties is a long and challenging process. Interestingly, we observed that MSC-CM contributes to the suppression of TRPM2 activation, indicating that MSCs secrete endogenous TRPM2 inhibitors. To identify such a component in the complex MSC-CM, we analyzed secreted protein expression and detected high levels many cytokines that may modulate the function of TRPM2 and warrant further investigation.

In eukaryotic cells, the MAPK signaling pathway regulates gene expression and processes such as cell proliferation, differentiation, apoptosis and stress response, and components of the pathway, including ERK, JNK, and p38, have been identified as therapeutic targets for many diseases[26-28]. Accumulating evidence supports a role for the MAPK signaling pathway in the pathogenesis and development of ischemic stroke[29, 30]. However, the upstream and downstream kinases of the MAPK signaling pathway are complex and have many influencing factors. Many studies have shown that the MAPK signaling pathway is the key modulator of ROS-related apoptosis[31, 32]. Therefore, we investigated the potential of MSC-CM to modulate the MAPK signaling pathway. Following treatment of HT22 cells with $\mathrm{H}_{2} \mathrm{O}_{2}$, expression levels of both p-ERK and p-JNK were decreased. We showed that subsequent MSC-CM treatment effectively prevented the $\mathrm{H}_{2} \mathrm{O}_{2}$-induced decrease in JNK expression, but had no effect on ERK expression. Furthermore, the protective effect of MSC-CM treatment against the $\mathrm{H}_{2} \mathrm{O}_{2}$-induced decrease in JNKs expression was accompanied by a decrease in neuronal cell apoptosis. These findings are consistent with previous reports that JNKs play roles in neuronal development and neurite growth[33, 34]. 
In this study, we evaluated the effects of UC-MSCs-CM on neuronal oxidative damage. We showed that UC-MSCs-CM protects neurons agains oxidative injury, possibly by inhibiting activation of the TRPM2 and JNK signaling pathway.

\section{Data accessibility statement}

The data used to support the findings of this study are included in this article.

\section{Competing interest}

The author(s) declared no potential conflicts of interest with respect to the research, authorship, and/or publication of this article.

\section{Funding}

This work was supported by the National Natural Science Foundation of China (No. 82060255), Key Research and Development Plan of Ningxia (No. 2019BFH02003, 2021BEG03098 and 2018BCG01002).

\section{Author contribution}

Xueyun Liang and Jianguo Niu contributed to the conception of the study and revised the article; Jianqiang Yu helped perform the analysis with constructive discussion; Yan Wang performed the main part of experiment and wrote the manuscript draft; Jiaxin Liu, Baocong Yu, Yiran Jin, Jiahui Li and Xiaona Ma performed the experimental work.

\section{Ethical approval}

All procedures performed in this study were approved and in accordance with the ethical standards of Ethics Committee of the General Hospital of Ningxia Medical University.

\section{References}

1. Manzanero S, Santro T and Arumugam TV (2013) Neuronal oxidative stress in acute ischemic stroke: sources and contribution to cell injury. Neurochem Int 62:712-8. doi: 10.1016/j.neuint.2012.11.009

2. Radak D, Resanovic I and Isenovic ER (2014) Link between oxidative stress and acute brain ischemia. Angiology 65:667-76. doi: 10.1177/0003319713506516

3. Wang T, Jian Z, Baskys A, Yang J, Li J, Guo H, Hei Y, Xian P, He Z, Li Z, Li N and Long Q (2020) MSC-derived exosomes protect against oxidative stress-induced skin injury via adaptive regulation of the NRF2 defense system. Biomaterials 257:120264. doi: 10.1016/j.biomaterials.2020.120264

4. Sayad Fathi S and Zaminy A (2017) Stem cell therapy for nerve injury. World J Stem Cells 9:144-151. doi: 10.4252/wjsc.v9.i9.144 
5. Dey R, Kemp K, Gray E, Rice C, Scolding N and Wilkins A (2012) Human mesenchymal stem cells increase anti-oxidant defences in cells derived from patients with Friedreich's ataxia. Cerebellum 11:861-71. doi: 10.1007/s12311-012-0406-2

6. Liu X, Zhou L, Chen X, Liu T, Pan G, Cui W, Li M, Luo ZP, Pei M, Yang H, Gong Y and $\mathrm{He} F$ (2016) Culturing on decellularized extracellular matrix enhances antioxidant properties of human umbilical cord-derived mesenchymal stem cells. Mater Sci Eng C Mater Biol Appl 61:437-48. doi: 10.1016/j.msec.2015.12.090

7. Park CM, Kim MJ, Kim SM, Park JH, Kim ZH and Choi YS (2016) Umbilical cord mesenchymal stem cell-conditioned media prevent muscle atrophy by suppressing muscle atrophy-related proteins and ROS generation. In Vitro Cell Dev Biol Anim 52:68-76. doi: 10.1007/s11626-015-9948-1

8. Ma N, Li S, Lin C, Cheng X and Meng Z (2021) Mesenchymal stem cell conditioned medium attenuates oxidative stress injury in hepatocytes partly by regulating the miR-4865p/PIM1 axis and the TGF-beta/Smad pathway. Bioengineered 12:6434-6447. doi: $10.1080 / 21655979.2021 .1972196$

9. Turlova E, Feng ZP and Sun HS (2018) The role of TRPM2 channels in neurons, glial cells and the blood-brain barrier in cerebral ischemia and hypoxia. Acta Pharmacol Sin 39:713721. doi: 10.1038/aps.2017.194

10. Belrose JC and Jackson MF (2018) TRPM2: a candidate therapeutic target for treating neurological diseases. Acta Pharmacol Sin 39:722-732. doi: 10.1038/aps.2018.31

11.Cruz-Torres I, Backos DS and Herson PS (2020) Characterization and Optimization of the Novel Transient Receptor Potential Melastatin 2 Antagonist tatM2NX. Mol Pharmacol 97:102-111. doi: 10.1124/mol.119.117549

12.Jiang LH, Yang W, Zou J and Beech DJ (2010) TRPM2 channel properties, functions and therapeutic potentials. Expert Opin Ther Targets 14:973-88. doi: 10.1517/14728222.2010.510135

13.Ye M, Yang W, Ainscough JF, Hu XP, Li X, Sedo A, Zhang XH, Zhang X, Chen Z, Li XM, Beech DJ, Sivaprasadarao A, Luo JH and Jiang LH (2014) TRPM2 channel deficiency prevents delayed cytosolic $\mathrm{Zn} 2+$ accumulation and CA1 pyramidal neuronal death after transient global ischemia. Cell Death Dis 5:e1541. doi: 10.1038/cddis.2014.494

14.Jia J, Verma S, Nakayama S, Quillinan N, Grafe MR, Hurn PD and Herson PS (2011) Sex differences in neuroprotection provided by inhibition of TRPM2 channels following experimental stroke. J Cereb Blood Flow Metab 31:2160-8. doi: 10.1038/jcbfm.2011.77

15.Alim I, Teves L, Li R, Mori Y and Tymianski M (2013) Modulation of NMDAR subunit 
expression by TRPM2 channels regulates neuronal vulnerability to ischemic cell death. J Neurosci 33:17264-77. doi: 10.1523/JNEUROSCI.1729-13.2013

16.Hack CT, Buck T, Bagnjuk K, Eubler K, Kunz L, Mayr D and Mayerhofer A (2019) A Role for H2O2 and TRPM2 in the Induction of Cell Death: Studies in KGN Cells. Antioxidants (Basel) 8. doi: 10.3390/antiox8110518

17.Gao M, Du Y, Xie JW, Xue J, Wang YT, Qin L, Ma MM, Tang YB and Li XY (2018) Redox signal-mediated TRPM2 promotes Ang II-induced adipocyte insulin resistance via $\mathrm{Ca}(2+)$-dependent $\mathrm{CaMKII/JNK}$ cascade. Metabolism 85:313-324. doi: 10.1016/j.metabol.2018.05.005

18.Bhang SH, Lee S, Shin JY, Lee TJ, Jang HK and Kim BS (2014) Efficacious and clinically relevant conditioned medium of human adipose-derived stem cells for therapeutic angiogenesis. Mol Ther 22:862-72. doi: 10.1038/mt.2013.301

19.Kay AG, Long G, Tyler G, Stefan A, Broadfoot SJ, Piccinini AM, Middleton J and Kehoe O (2017) Mesenchymal Stem Cell-Conditioned Medium Reduces Disease Severity and Immune Responses in Inflammatory Arthritis. Sci Rep 7:18019. doi: 10.1038/s41598-01718144-w

20.Huang W, Lv B, Zeng H, Shi D, Liu Y, Chen F, Li F, Liu X, Zhu R, Yu L and Jiang X (2015) Paracrine Factors Secreted by MSCs Promote Astrocyte Survival Associated With GFAP Downregulation After Ischemic Stroke via p38 MAPK and JNK. J Cell Physiol 230:2461-75. doi: 10.1002/jcp.24981

21. Tsai MJ, Tsai SK, Hu BR, Liou DY, Huang SL, Huang MC, Huang WC, Cheng H and Huang SS (2014) Recovery of neurological function of ischemic stroke by application of conditioned medium of bone marrow mesenchymal stem cells derived from normal and cerebral ischemia rats. J Biomed Sci 21:5. doi: 10.1186/1423-0127-21-5

22.Mai C, Mankoo H, Wei L, An X, Li C, Li D and Jiang LH (2020) TRPM2 channel: A novel target for alleviating ischaemia-reperfusion, chronic cerebral hypo-perfusion and neonatal hypoxic-ischaemic brain damage. J Cell Mol Med 24:4-12. doi: 10.1111/jcmm. 14679

23.Shimizu T, Dietz RM, Cruz-Torres I, Strnad F, Garske AK, Moreno M, Venna VR, Quillinan N and Herson PS (2016) Extended therapeutic window of a novel peptide inhibitor of TRPM2 channels following focal cerebral ischemia. Exp Neurol 283:151-6. doi: 10.1016/j.expneurol.2016.06.015

24.Akpinar H, Naziroglu M, Ovey IS, Cig B and Akpinar O (2016) The neuroprotective action of dexmedetomidine on apoptosis, calcium entry and oxidative stress in cerebral ischemia-induced rats: Contribution of TRPM2 and TRPV1 channels. Sci Rep 6:37196. doi: 
$10.1038 /$ srep37196

25.Zhang H, Yu P, Lin H, Jin Z, Zhao S, Zhang Y, Xu Q, Jin H, Liu Z, Yang W and Zhang L (2021) The Discovery of Novel ACA Derivatives as Specific TRPM2 Inhibitors that Reduce Ischemic Injury Both In Vitro and In Vivo. J Med Chem 64:3976-3996. doi: 10.1021/acs.jmedchem.0c02129

26.Zheng Y, Han Z, Zhao H and Luo Y (2020) MAPK: A Key Player in the Development and Progression of Stroke. CNS Neurol Disord Drug Targets 19:248-256. doi: $10.2174 / 1871527319666200613223018$

27.Irving EA and Bamford M (2002) Role of mitogen- and stress-activated kinases in ischemic injury. J Cereb Blood Flow Metab 22:631-47. doi: 10.1097/00004647-20020600000001

28.Sun J and Nan G (2016) The Mitogen-Activated Protein Kinase (MAPK) Signaling Pathway as a Discovery Target in Stroke. J Mol Neurosci 59:90-8. doi: 10.1007/s12031-0160717-8

29.Kyriakis JM and Avruch J (2012) Mammalian MAPK signal transduction pathways activated by stress and inflammation: a 10-year update. Physiol Rev 92:689-737. doi: 10.1152/physrev.00028.2011

30.Zhang J, Li Y, Wang C, Wang Y, Zhang Y, Huang L and Zhang Z (2020) Lysophosphatidic Acid Induces Apoptosis of PC12 Cells Through LPA1 Receptor/LPA2 Receptor/MAPK Signaling Pathway. Front Mol Neurosci 13:16. doi: $10.3389 /$ fnmol.2020.00016

31.Martinez MA, Ubeda A and Trillo MA (2021) Role of NADPH oxidase in MAPK signaling activation by a $50 \mathrm{~Hz}$ magnetic field in human neuroblastoma cells. Electromagn Biol Med 40:103-116. doi: 10.1080/15368378.2020.1851250

32.Cao X, Fu M, Bi R, Zheng X, Fu B, Tian S, Liu C, Li Q and Liu J (2021) Cadmium induced BEAS-2B cells apoptosis and mitochondria damage via MAPK signaling pathway. Chemosphere 263:128346. doi: 10.1016/j.chemosphere.2020.128346

33.Atkinson PJ, Cho CH, Hansen MR and Green SH (2011) Activity of all JNK isoforms contributes to neurite growth in spiral ganglion neurons. Hear Res 278:77-85. doi: 10.1016/j.heares.2011.04.011

34.Kuan CY, Yang DD, Samanta Roy DR, Davis RJ, Rakic P and Flavell RA (1999) The Jnk1 and Jnk2 protein kinases are required for regional specific apoptosis during early brain development. Neuron 22:667-76. doi: 10.1016/s0896-6273(00)80727-8 


\section{Figure Captions}

Figure 1. UC-MSCs-CM protected HT22 cells against $\mathrm{H}_{2} \mathrm{O}_{2}$-induced neuronal apoptosis. A: Representative images of HT22 cell morphology, scale bar $=100 \mu \mathrm{m}$; B: Representative images of immunofluorescence staining of cleaved Caspase-3, scale bar $=20 \mu \mathrm{m}$; C: Percentages of the Caspase-3-positive cells; D: Representative images of Western blot analysis of cleaved-Caspase-9; E: Relative expression analysis of cleaved-Caspase-9. Data represent the mean \pm SD. ${ }^{*} P<0.05$ compared with Normal group, ${ }^{\#} P<0.05$ compared with MSCs-CM group.

Figure 2. UC-MSC-CM is composed of cellular growth factors and possessed antioxidative ability. A: ELISA analysis of the concentration of various factors in the supernatant of MSCs$\mathrm{CM}$ and fibroblast-CM; B: Representative images of Western blot analysis of SOD; C: Relative expression of SOD. Data represent the mean \pm SD. ${ }^{*} P<0.05$ compared with Normal group, ${ }^{\#} P<0.05$ compared with MSCs-CM group.

Figure 3. UC-MSC-CM suppressed TRPM2-related currents. A: Representative images of immunofluorescence staining of TRPM2, scale bar $=20 \mu \mathrm{m}$.; B: I-V curve of TRPM2 showing changes in membrane potential.

Figure 4. UC-MSCs-CM protection of HT22 cells was mediated via the JNK signaling pathway. A: Representative images of the Western blot analysis of the protein expression of pJNK1/2/3 protein expression; B: Relative expression of $\mathrm{p}-\mathrm{JNK} 1 / 2 / 3$; C: Representative images of the Western blot analysis of cleaved-Caspase-9 protein expression; D: Relative expression of cleaved-Caspase 9; E: Representative images of the Western blot analysis of p-ERK1/2 protein; F: Relative expression of $\mathrm{p}$-ERK1/2. Data represent the mean $\pm \mathrm{SD} .{ }^{*} P<0.05$ compared with Normal group, ${ }^{\#} P<0.05$ compared with MSCs-CM group. 


\section{Figures}

\section{Figure 1}

UC-MSCs-CM protected HT22 cells against H2O2-induced neuronal apoptosis. A: Representative images of HT22 cell morphology, scale bar $=100 \mu \mathrm{m}$; B: Representative images of immunofluorescence staining of cleaved Caspase-3, scale bar $=20 \mu \mathrm{m}$; C: Percentages of the Caspase-3-positive cells; D:

Representative images of Western blot analysis of cleaved-Caspase-9; E: Relative expression analysis of cleaved-Caspase-9. Data represent the mean \pm SD. ${ }^{*} P<0.05$ compared with Normal group, \# $P<0.05$ compared with MSCs-CM group.

\section{Figure 2}

UC-MSC-CM is composed of cellular growth factors and possessed antioxidative ability. A: ELISA analysis of the concentration of various factors in the supernatant of MSCsCM and fibroblast-CM; $\mathrm{B}$ : Representative images of Western blot analysis of SOD; C: Relative expression of SOD. Data represent the mean $\pm S D$. ${ }^{*} P<0.05$ compared with Normal group, \# $P<0.05$ compared with MSCs-CM group.

\section{Figure 3}

UC-MSC-CM suppressed TRPM2-related currents. A: Representative images of immunofluorescence staining of TRPM2, scale bar $=20 \mu \mathrm{m}$.; B: I-V curve of TRPM2 showing changes in membrane potential.

\section{Figure 4}

UC-MSCs-CM protection of HT22 cells was mediated via the JNK signaling pathway. A: Representative images of the Western blot analysis of the protein expression of $p \mathrm{JNK} 1 / 2 / 3$ protein expression; $\mathrm{B}$ : Relative expression of p-JNK1/2/3; C: Representative images of the Western blot analysis of cleavedCaspase-9 protein expression; D: Relative expression of cleaved-Caspase 9; E: Representative images of the Western blot analysis of $p$-ERK1/2 protein; F: Relative expression of $p$-ERK1/2. Data represent the mean \pm SD. ${ }^{*} \mathrm{P}<0.05$ compared with Normal group, \# $\mathrm{P}<0.05$ compared with MSCs-CM group. 